\title{
Curtailment Estimation Methods for Demand Response
}

\author{
Lessons Learned by Comparing Apples to Oranges \\ Charalampos Chelmis, Muhammad Rizwan Saeed, Marc Frincu, Viktor K. Prasanna \\ Ming Hsieh Electrical Engineering Department \\ University of Southern California \\ \{chelmis, saeedm, frincu, prasanna\}@usc.edu
}

\begin{abstract}
Accurate estimation and evaluation of consumption reduction achieved by participants during Demand Response is critical to Smart Grids. We perform an in-depth study of popular estimation methods used to determine the extent of consumption shedding during DR, using a real-world Smart Grid dataset from the University of Southern California campus microgrid. We provide insights to the process of selecting a reasonable baseline with respect to potential misinterpretation of the estimation of electricity consumption reduction during DR.
\end{abstract}

\section{Categories and Subject Descriptors}

G.3 [Probability and Statistics]: Time Series Analysis; H.4.m [Information Systems Applications]: Miscellaneous

\section{Keywords}

Baseline Models; Reduced Consumption; Load Forecasting

\section{INTRODUCTION}

In this work, we statistically analyze the effect of Baseline Load Profile (BLP) models on the interpretation of consumption reduction as a result of Demand Response (DR) $[6,10]$ using real-world data from the University of Southern California (USC) microgrid, with the objective of improving the accuracy of estimating electricity demand reduction due to participation in DR programs. Accurate estimation and evaluation of consumption reduction achieved by participants during curtailment is critical to DR programs [6], particularly when participation is voluntary [1]. The amount of computed curtailment depends on the accuracy of the baseline model used. As many baseline models exist, different curtailment estimates can be derived. The problem with calculating BLP model accuracy, lies mainly in the fact that there is no actual reference value to compare against. We argue that without careful consideration, utility providers can

Permission to make digital or hard copies of part or all of this work for personal or classroom use is granted without fee provided that copies are not made or distributed for profit or commercial advantage and that copies bear this notice and the full citation on the first page. Copyrights for third-party components of this work must be honored. For all other uses, contact the Owner/Author. Copyright is held by the owner/author(s). e-Energy'15, July 14-17, 2015, Bangalore, India. ACM 978-1-4503-3609-3/15/07.

http://dx.doi.org/10.1145/2768510.2775332 . end up with erroneous data on the actual curtailment which can in turn lead to billing or rewarding issues. We show that choosing a good baseline depends on both intrinsic (e.g., DR strategy, day of week) and extrinsic (e.g., temperature, human behavior) factors. To the best of our knowledge, our work is the first to provide an in-depth comparative analysis of the effect of BLP models for post DR analysis in a real-world, large-scale setting.

\section{REAL-WORLD CASE STUDY}

We consider a real-world Smart Grid dataset from the University of Southern California campus microgrid ${ }^{1}$. The dataset comprises of a collection of observed electricity consumption values (measured in $\mathrm{kWh}$ at every 15 minutes) from 35 diverse buildings, collected over a one year period (November 2012 - December 2013) [3]. Using our real-world dataset, we benchmark a set of BLPs: Auto Regressive Integrated Moving-Average (ARIMA) [5], New York ISO (NYISO) [8], Southern California Edison ISO (CASCE) [9], California ISO (CAISO) [2] and a modified version that introduces a morning adjustment factor (CAISOm) [6], and Fixed Value (i.e., the consumption value just prior to the beginning of the DR event is used as the predictor).

We examine the performance of a baseline in terms of bias, i.e., dominance of positive or negative predictions, and accuracy, i.e., average absolute percent error. To measure model bias, we measure the median of the distribution of errors. Intuitively, the closest to zero the median of the error is, the more unbiased the model. We measure average deviance between predicted consumption, $f c_{t}^{15}$, and actual consumption, $a c_{t}^{15}$, on non-DR days (between $1-5 \mathrm{pm}$, for consistency with DR days), as $M P E=\frac{100}{n} \sum_{t=1}^{n} \frac{f c_{t}^{15}-a c_{t}^{15}}{a c_{t}^{15}}$. We found CASCE to perform the best among all BLPs, achieving good MAPE values while at the same time being the least biased.

\section{ACHIEVING A CURTAILMENT GOAL}

To shed light on the effect of baseline selection on the interpretation of consumption reduction estimation and evaluation due to DR programs, we consider DR events in which all buildings participate, each following a random DR strategy (e.g., Duty Cycling, Variable Frequency Drive [7], Global Temperature Reset [7]). In order to ensure that randomly selecting a strategy for each building does not affect our findings, we repeated the experiment, with the difference that the "best" strategy per building was used. Evidently, curtailment estimation is highly correlated to the baseline selected

\footnotetext{
${ }^{1}$ The dataset is available upon request for academic use from the USC Facility Management Services (FMS).
} 


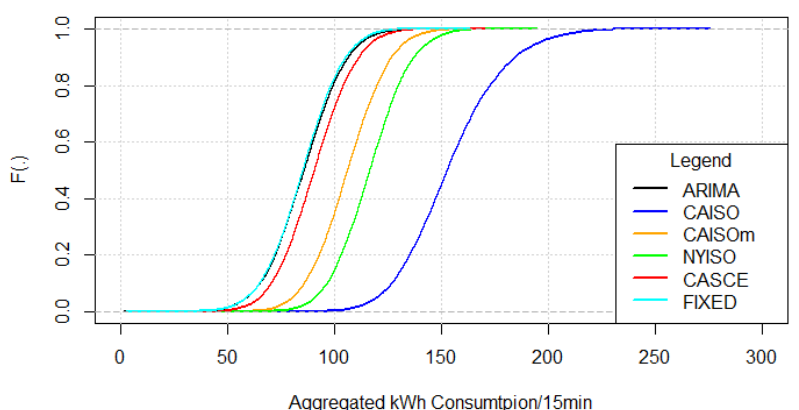

(a) Probability density function

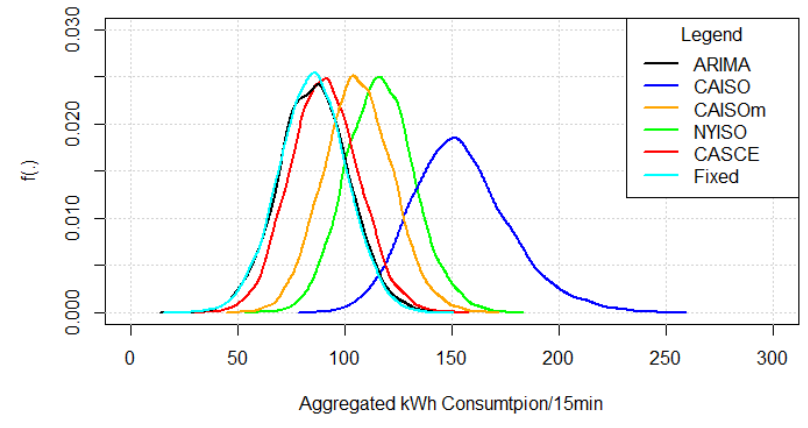

(b) Cumulative density function

Figure 1: Aggregate curtailment over all buildings.

for analysis. Therefore more effort should be allocated in the following areas of research. First, better baseline methods that can be applied to all customers without exhibiting volatility to external factors would be highly desirable. If a "one solution fits all" is not possible, developing a framework that would adapt to individual household attributes so as to select the "best" performing baseline method for each individual customer would be advisable. Learning to switch between baselines as time progresses to adapt to customers (changing) behavior would also be beneficial, but at the same time computationally expensive.

Instead of estimating what the consumption would have been in the absence of DR (i.e., baseline consumption), and then calculating the difference between such estimate and the actual consumption during DR, computational methods for reduced consumption prediction would be beneficial. The advantage of such an approach is twofold. First, reduced consumption prediction does not require a baseline calculation. Instead, observed curtailed consumption from past events could be used to predict future curtailed consumption. Second, predicted values would be directly comparable against observed consumption during DR for a fair performance evaluation. Some works [4] have already proposed solutions towards this direction. Our findings motivate an exploration of promising future work.

The drawback of our work is that it only considers a single regional scenario, even though our analysis involves a heterogeneous collection of buildings with diverse functions and purpose, covering a wide percentage of consumer demographics. Considering scenarios on a per-household basis, as well as including more diverse customer types (e.g. industrial or residential) would strengthen our study.

\section{Acknowledgments}

This material is based upon work supported by the United States Department of Energy under Award Number number DE-OE0000192, and the Los Angeles Department of Water and Power (LA DWP). The views and opinions of authors expressed herein do not necessarily state or reflect those of the United States Government or any agency thereof, the LA DWP, nor any of their employees.

\section{REFERENCES}

[1] S. Aman, Y. Simmhan, and V. K. Prasanna. Energy management systems: state of the art and emerging trends. IEEE Communications Magazine, 51(1):114-119, 2013.

[2] CaliforniaISO. Caiso demand response resource user guide. Technical report, 2007. http://www.caiso.com/1ca6/1ca67a5816ee0.pdf.

[3] C. Charalampos, S. Muhammad Rizwan, F. Marc, and P. Viktor K. Curtailment estimation methods for demand response. Technical report, University of Southern California, 2015.

[4] C. Chelmis, S. Aman, M. R. Saeed, M. Frincu, and V. K. Prasanna. Estimating reduced consumption for dynamic demand response. In Proceedings of the Twenty-Ninght AAAI Conference on Artificial Intelligence. AAAI Press, 2015.

[5] B. George E. P. and J. Gwilym M. Time series analysis, forecasting and control. Holden-Day, 1970.

[6] C. Katie, P. Mary Ann, G. Charles A., and K. Sila. Statistical analysis of baseline load models for non-residential buildings. Energy and Buildings, 41(4):374-381, 2009.

[7] N. Motegi, M. A. Piette, D. S. Watson, S. Kiliccote, and $\mathrm{P}$. Xu. Introduction to commercial building control strategies and techniques for demand response. Technical report, Lawrence Berkely National Laboratory, 2006. http://gaia.lbl.gov/btech/papers/59975.pdf.

[8] NewYorkISO. Emergency demand response program manual. Technical report, 2013. http://www.nyiso.com/public/webdocs/markets \ _operations/documents/Manuals\_and \_Guides/ Manuals/Operations/edrp\_mnl.pdf.

[9] SouthernCaliforniaEdison. 10 day average baseline and "day-off" adjustment. Technical report, 2011. http://asset.sce.com/Documents/Business - Energy Management Solutions/10DayAvgBaselineFS.pdf.

[10] A. Veit, C. Goebel, R. Tidke, C. Doblander, and H. Jacobsen. Household electricity demand forecasting: benchmarking state-of-the-art methods. In The Fifth International Conference on Future Energy Systems, e-Energy '14, Cambridge, United Kingdom June 11 - 13, 2014, pages 233-234. ACM, 2014. 